\title{
Sesamin induces autophagy in colon cancer cells by reducing tyrosine phosphorylation of EphA1 and EphB2
}

\author{
HIROMI TANABE*, KAGEAKI KURIBAYASHI*, NAOKI TSUJI, \\ MAKI TANAKA, DAISUKE KOBAYASHI and NAOKI WATANABE \\ Department of Clinical Laboratory Medicine, Sapporo Medical University \\ School of Medicine, South-1, West-16, Chuo-ku, Sapporo 060-8543, Japan
}

Received January 14, 2011; Accepted March 29, 2011

DOI: 10.3892/ijo.2011.1011

\begin{abstract}
Receptor tyrosine kinase EphB2 and autophagic machinery are known as tumor suppressors; however, the connection remains to be elucidated. Here, we show the link between EphB2 and autophagy. Sesamin, a major lignan in sesame oil, induced autophagy in the human colon cancer cell lines HT29 and LS180, as shown by electron microscopy, as well as Western blotting and immunofluorescence imaging using an anti-LC3 antibody. Receptor tyrosine kinase array analysis revealed that sesamin treatment increased the levels of unphosphorylated -EphA1 and -EphB2 in HT29 cells. Silencing of EphA1 and EphB2 blocked sesamin-induced autophagy as well as sesamin-induced loss of cell viability. These results show that EphA1 and EphB2 play a critical role in this process. The present study reveals a novel function for EphA1 and EphB2 in the induction of autophagy, suggesting a tumor suppressor role for these proteins in colorectal cancer.
\end{abstract}

\section{Introduction}

Sesamin is a major lignan constituent of sesame oil. Sesamin isolated from the oil of sesame seeds (Sesamum indicum) has been associated with prevention of hypertension (1), thrombogenesis (2), and hypercholesteremia by increasing hepatic fatty acid oxidation $(3,4)$. In addition, sesamin exhibits antioxidative properties by reducing peroxidation products in the plasma and liver of rats. Sesamin has also been shown to have an inhibitory effect on chemically induced cancers. Sesamin inhibits the growth of a variety of neoplastic cells, including leukemia, multiple myeloma, and cancers of the colon, prostate, pancreas and lung $(5,6)$. However, the mechanisms mediating

Correspondence to: Dr Naoki Watanabe, Department of Clinical Laboratory Medicine, Sapporo Medical University School of Medicine, South-1, West-16, Chuo-ku, Sapporo 060-8543, Japan

E-mail:watanabn@sapmed.ac.jp

*Contributed equally

Key words: sesamin, autophagy, EphA1, EphB2, colorectal cancer, receptor tyrosine kinase the effect of sesamin on the inhibition of malignant cell growth remain to be elucidated.

Autophagy is one of the most important protein degradation systems in eukaryotic cells, and is mainly responsible for the degradation of long-lived proteins and cellular organelles (7). There are 3 types of autophagy that differ in the mode of delivery of cargo to lysosomes, namely macroautophagy, microautophagy, and chaperone-mediated autophagy. Macroautophagy, which will be referred to as autophagy in the present study, is a bulk degradation system usually activated in response to metabolic stress, including deprivation of nutrients, oxygen, or growth factors as well as high temperature. Intracellular stresses such as damaged organelles, accumulation of mutant proteins and microbial invasion activate the autophagy pathway $(8,9)$.

Dysregulation of autophagy leads to various human diseases, including neurodegenerative disorders and cancer. In general, autophagic capacity of cancer cells is lower than their normal counterpart (10-14). In experimental animal model, cells from neoplastic nodules of the liver showed decreased autophagic potential than normal cells from the liver (15-17). Beclin-1, an essential signaling molecule in the autophagy pathway, is localized to chromosome $17 \mathrm{q} 21$, a locus deleted in $75 \%$ of ovarian, $50 \%$ of breast, and $40 \%$ of prostate cancer (18). Moreover, heterozygous deletion of beclin- 1 in mice causes high incidences of spontaneous lung adenocarcinoma, hepatocellular carcinoma and lymphoma $(19,20)$. Tumor suppressor genes that are frequently mutated in human cancers (PTEN and p53) turn autophagy on and oncogenic signals (Ras, PI3 kinase, Akt) turn it off $(18,21)$. Furthermore, net deletions of several autophagyspecific genes are commonly found in human malignancies (22). These observations indicate that autophagy is a tumor suppressor mechanism.

In the present study, we demonstrate that sesamin inhibits the growth of colon cancer cell lines by triggering autophagy and show the essential role of the ephrin receptors EphA1 and EphB2 in this process.

\section{Materials and methods}

Reagents. Sesamin was purchased from Cayman Chemical Company (Ann Arbor, MI). Anti-LC3 antibodies for immunofluorescence staining and Western blotting were purchased from MBL (Nagoya, Japan) and Cell Signaling Technology 
(Danvers, MA), respectively. Anti-EphB2 antibody was purchased from R\&D Systems (Minneapolis, NE). Anticleaved PARP antibody was purchased from Cell Signaling Technology. Anti-HMGB1 antibody was purchased from Abcam (Cambridge, MA).

Cell culture. The human colon cancer cell lines HT29 and LS180 were obtained from American Type Culture Collection (Manassas, VA). The cell lines were cultured in Dulbecco's modified Eagle's medium (DMEM; Sigma-Aldrich, St. Louis, MO) supplemented with $10 \%$ fetal bovine serum (FBS; Hyclone Thermo Fischer Scientific, Logan, UT) in humidified $5 \% \mathrm{CO}_{2}$ at $37^{\circ} \mathrm{C}$.

Measurement of cell viability by ATP assay. The CellTiter-Glo Luminescent Cell Viability Assay (Promega, Madison, WI) was used according to the manufacturer's instructions. Briefly, 1,000 cells were seeded in each 96-well culture plates (Corning, Corning, NY) in $100 \mu 1$ DMEM supplemented with 10\% FBS. Cells were treated with various concentrations of sesamin and incubated for $72 \mathrm{~h}$, after which the assay reagent was added and incubated for $10 \mathrm{~min}$ at room temperature (RT). The luminescent signal was measured using a Veritas Microplate Luminometer (Promega Madison, WI).

Cell cycle analysis. After the indicated treatments, the cells were collected and fixed with $70 \%$ ethanol at $4^{\circ} \mathrm{C}$. The samples were suspended in phosphate-citrate buffer. After centrifugation, the pellets were treated with RNase A, stained with propidium iodide (PI), and analyzed by flow cytometry (EPICS XL-MCL cytometer; Beckman Coulter, Fullerton, CA).

Electron microscopy. After the indicated treatments, the cells were washed and fixed with $2.5 \%$ gutaraldehyde at $4^{\circ} \mathrm{C}$ until they were embedded. The samples were postfixed with $1 \%$ osmium tetroxide for $2 \mathrm{~h}$ at $4^{\circ} \mathrm{C}$. Cells were postfixed with $2 \%$ osmium tetroxide followed by dehydration using an increasing gradient of ethanol and propylene oxide, after which the samples were embedded in Quetol 812 (Nissin EM). Ultrathin sections were stained with uranyl acetate and lead citrate and examined with an H7500 electron microscope (Hitachi, Tokyo, Japan).

Immunofluorescent staining for $L C 3$. After the various treatments indicated in each figure, cells were fixed in $4 \%$ paraformaldehyde in phosphate-buffered saline (PBS) for $15 \mathrm{~min}$ at RT, washed, and permeabilized with $0.1 \%$ Triton $\mathrm{X}$ in PBS for $10 \mathrm{~min}$ at RT. The cells were incubated with anti-LC3 antibody (1/200 dilution) for $24 \mathrm{~h}$ at $4^{\circ} \mathrm{C}$, washed, and incubated with anti-rabbit IgG Alexa Flour 488 antibody (1/1,000 dilution) (Invitrogen, Carlsbad, CA) for $1 \mathrm{~h}$ at RT. Finally, the samples were imaged using a confocal laser scanning microscope (Radian; Carl Zeiss, Thornwood, NY).

Western blotting. Western blotting was carried out by standard methods. Briefly, cells were sonicated in lysis buffer $[20 \mathrm{mM}$ Tris-HCl (pH 8.0), 137 mM NaCl, 5 mM EDTA, 1\% NP-40, $1 \mathrm{mM}$ sodium orthovanadate, $10 \mu \mathrm{g} / \mathrm{ml}$ aprotinin, $10 \mu \mathrm{g} / \mathrm{ml}$ leupeptin] with a protease inhibitor cocktail (Sigma-Aldrich). The samples were separated on a Tris-Glycine gel (Invitrogen) under denaturing conditions. Proteins were electro-blotted onto a nitrocellulose membrane. After incubation with the indicated antibodies, the proteins were detected using ECL Western Blotting Detection kit (GE Healthcare, Piscataway, $\mathrm{NJ}$ ). The blots were visualized using the ChemiDoc XRS system (Bio-Rad Laboratories, Hercules, CA).

Measurement of caspase- 3 and -7 activities. Caspase- 3 and -7 activities were measured using Caspase-Glo 3/7 Assay (Promega). Five thousand cells were seeded in each well of 96-well plates, and the cells were treated with sesamin. The assay reagent $(100 \mu \mathrm{l})$ was added to each well and incubated for $1 \mathrm{~h}$ at RT. The luminescence signal was measured using a Veritas $^{\mathrm{TM}}$ Microplate Luminometer.

Measurement of early apoptosis. Apoptotic cells were analyzed using the MEBCyto-Apoptosis kit (Medical \& Biological Laboratories, Nagaya, Japan), which is a combination of Annexin V and PI staining. After HT29 and LS180 cells were treated with vehicle or $50 \mu \mathrm{M}$ sesamin for the times indicated, cells were collected, incubated in a solution containing Annexin V-FITC and PI for 15 min, and analyzed by flow cytometry.

Analysis of senescent cells. The senescence-associated $\beta$-Gal activity was measured using a senescence detection kit (Calbiochem, San Diego, CA) according to the manufacturer's instructions. Stained cells were counted using an Olympus fluorescence microscope and the percentage of positive cells are shown as bar graphs.

Receptor tyrosine kinase (RTK) array analysis. The phosphorylation status of RTKs was analyzed using a human Phospho-RTK Array (R\&D Systems), which includes 42 different RTKs, following the manufacturer's instructions. Briefly, HT29 cells were treated with $50 \mu \mathrm{M}$ sesamin or vehicle for $72 \mathrm{~h}$. The samples were sonicated in lysis buffer on ice. Protein concentration was determined using the Dc Protein Assay kit (Bio-Rad Laboratories) according to the manufacturer's instructions. The array membranes were blocked and incubated with $500 \mu \mathrm{g}$ of cell lysate overnight at $4^{\circ} \mathrm{C}$ on a rocking platform shaker. Then, the array membranes were washed, incubated with HRP-conjugated anti-phosphotyrosine antibody for $2 \mathrm{~h}$ at RT, washed, and developed using ECL detection reagent (Amersham Pharmacia Biotec, Piscataway, NJ). The chemiluminescent signals were measured in duplicate using the ChemiDoc XRS system (Bio-Rad Laboratories), and the densitometric units were normalized against positive control spots on the same membrane provided by the manufacturer.

Transduction of short interfering RNA (siRNA). siRNAs were transfected into cells by electroporation, using Nucleofector II (Amaxa Biosystems, Gaithersburg, MD) according to the manufacturer's instructions. The programs W-017 and T-020 were used for HT29 and LS180 cells, respectively. The sequences 5'-GCAUGAACUACCUCAGUA-3' and 5'-CCAUCAAGCU CUACUGUAA-3' were used to silence EphA1 and EphB2, respectively. The sequence 5'-UAGCGACUAAACACAUCA AUU-3' was used as a non-silencing control. These siRNAs were synthesized and purchased from Sigma-Aldrich Japan (Ishikari, Japan). 
A
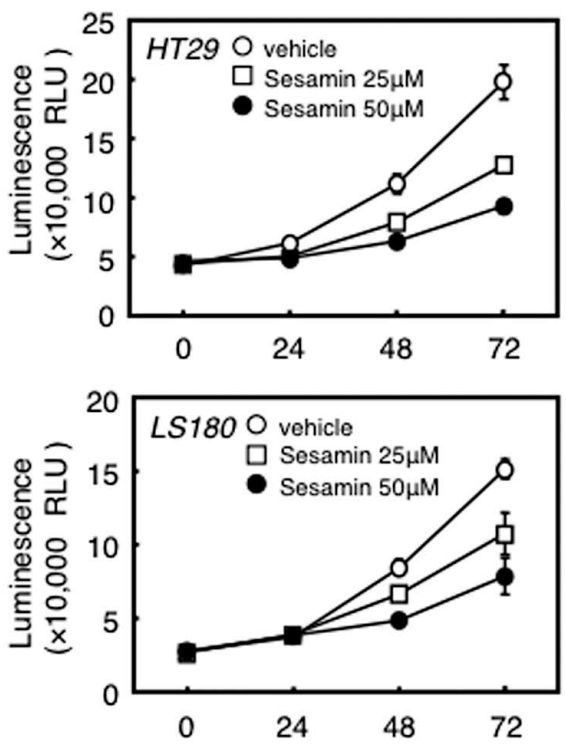

Time $(h)$
B

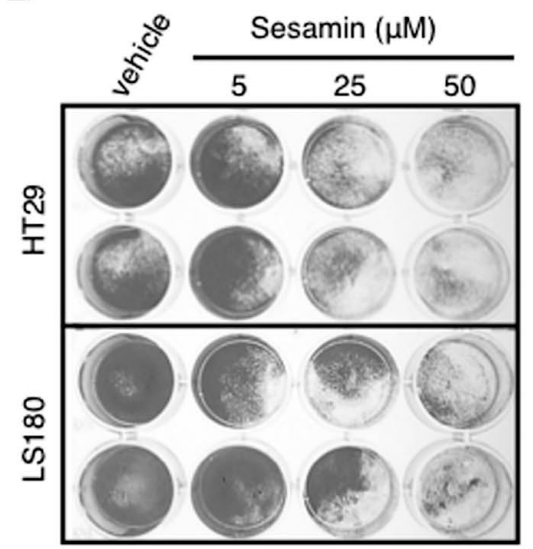

C

Sesamin exposure time $(h)$

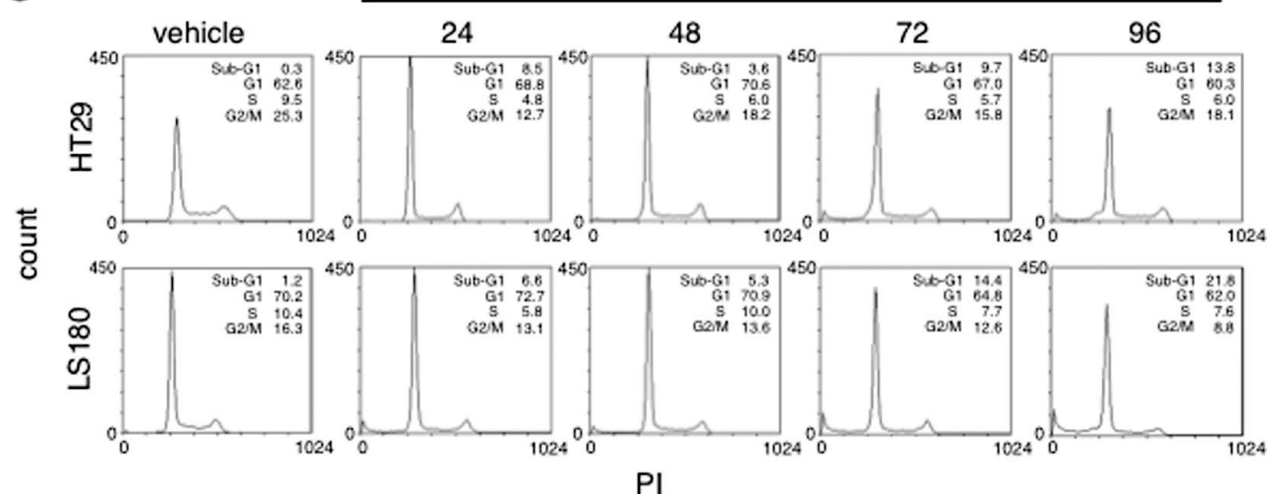

Figure 1. Sesamin inhibits cell viability of colon cancer cells. (A) HT29 and LS180 cells were seeded in 96-well plates in quadruplicate and treated with vehicle or sesamin for up to $72 \mathrm{~h}$. Cell viability was measured by ATP assay as described in Materials and methods. (B) HT29 and LS180 cells were seeded in 24-well plates and treated with vehicle or sesamin for 1 week. The complete medium with or without sesamin was replaced every $48 \mathrm{~h}$. At the end of the assay, the cells were stained with Coomassie Brilliant Blue as shown. (C) HT29 and LS180 cells were treated with vehicle or $50 \mu$ M sesamin for the times indicated in the figure. The cell cycles were analyzed as described in Materials and methods.

Quantification of EphAl and EphB2 mRNA. The expression of EphA1 and EphB2 mRNA was determined by quantitative reverse transcriptase polymerase chain reaction (RT-PCR) using ABI PRISM 7700 sequence detector system (Applied Biosystems, Foster City, CA). Total RNA was isolated using the RNeasy Plus Mini kit (Qiagen, Valencia, CA) according to the manufacturer's instructions. The cDNA was reverse transcribed using Taqman Reverse Transcription Reagents (Applied Biosystems, Branchburg, NJ). The gene-specific primers and fluorescent hybridization probes were as follows. For EphA1 mRNA, 5'-AACCTGCCAGACTAGGCTATCG-3', 5'-ACCC CCACCTCCCTTTTAAA-3' and 5'-(FAM) TGCTCTGCAC CCCAGAAAACCTCTTTG (TAMRA)-3', were used as forward primer, reverse primer, and the TaqMan probe, respectively. For EphB2, 5'-AGACCATGACAGAAGCCGAG TAC-3',5'-CAACCACAGCAATGAGGAAGAC-3' and 5'-(FAM) AAGCATCCAGGAGAAGTTGCCACTCATC (TAMRA)-3' were used as forward primer, reverse primer, and the TaqMan probe, respectively. The amounts of mRNA were normalized to the ratios of GAPDH. PCR primers and the probe for GAPDH were purchased from Applied Biosystems.

\section{Results}

Sesamin reduces cell viability in colon cancer cell lines. To examine the antitumor effect of sesamin in colon cancer cells, HT29 and LS180 cells were treated with various concentrations of sesamin up to 72 h. As shown in Fig. 1A, sesamin inhibited cell viability in a dose- and time-dependent manner. A longterm assay also showed growth inhibition of cells treated with sesamin (Fig. 1B). Further investigation into the mechanism of sesamin-mediated inhibition of cell growth showed that sesamin had no apparent effect on cell cycle (Fig. 1C).

Sesamin induces autophagy in colon cancer cells. To further investigate the mecahism how sesamin reduces cell viability 
A
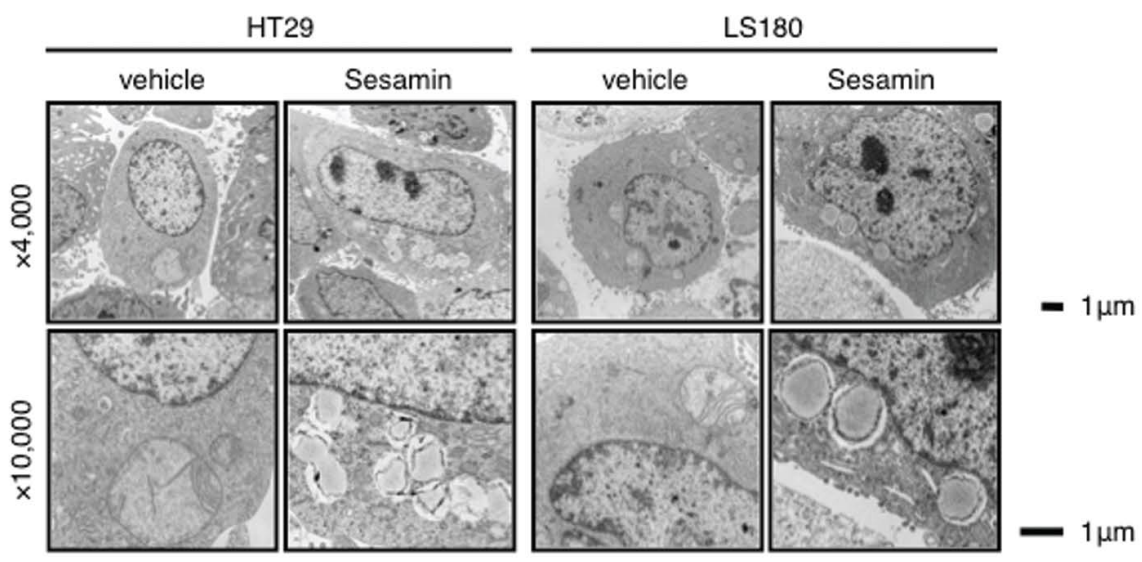

B

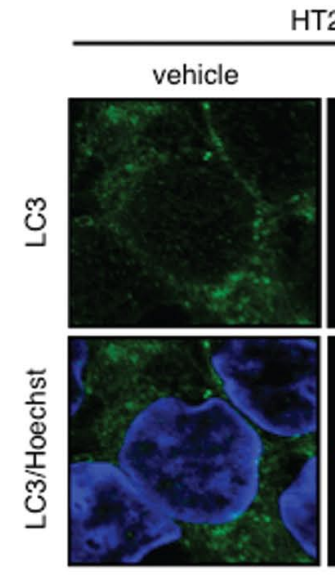

HT29

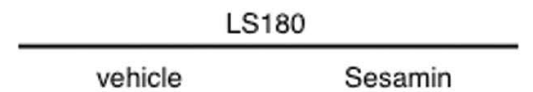

C

D
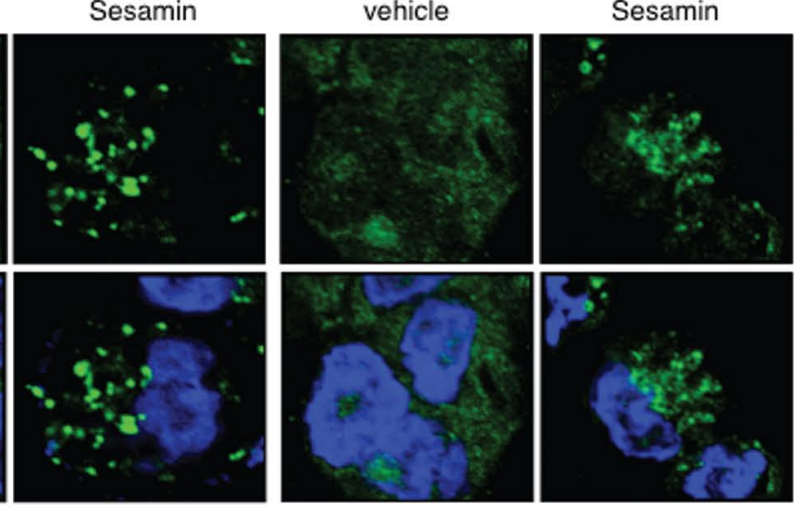

$E$
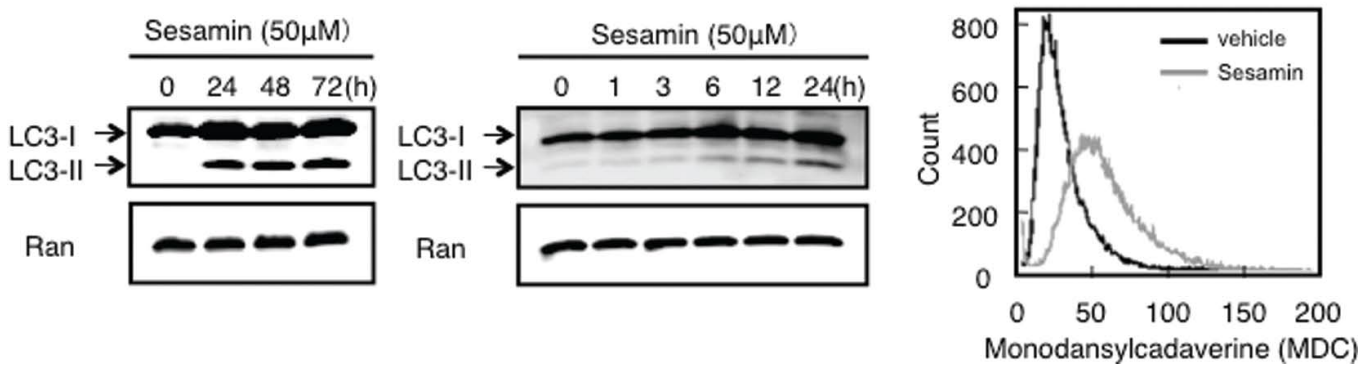

Figure 2. Sesamin induces autophagy in colon cancer cells. (A and B) HT29 and LS180 cells were treated with vehicle or $50 \mu \mathrm{M}$ sesamin for $72 \mathrm{~h}$. The images show electron microscopy (A) and immunofluorescence study using anti-LC3 antibody (B). For the immunofluorescence study, Hoechst 33342 was used to counter stain the nuclei. (C and D) HT29 cells were treated with $50 \mu \mathrm{M}$ sesamin for the times indicated. The results of the Western blot analysis using an anti-LC3 antibody are shown. (E) HT29 cells treated with or without $50 \mu \mathrm{M}$ sesamin for $72 \mathrm{~h}$. Autophagic cells were quantified by MDC staining as described in Materials and methods.

of colon cancer cells, electron-microscopy studies were carried out, and the results showed prominent double-membrane vacuoles engulfing cytosolic components in sesamin-treated cells (Fig. 2A). Cells showing the characteristic signs of necrosis or apoptosis were not detected. As shown in Fig. 2B, in cells treated with vehicle, LC-3 was distributed in the cytosol, while sesamin treatment resulted in the detection of LC3 punctate structures. The results of the Western blot analysis using an anti-LC3 antibody were consistent with the immunofluorescence study and showed that sesamin treatment induced LC3-II conversion in a time-dependent manner (Fig. 2C and D). Because monodansylcadaverine (MDC) accumulates in mature autophagic vacuoles, MDC staining can be used to detect the presence of these vacuoles (23). As shown in Fig. 2E, sesamin treatment caused an increase MDC-positive cells.

Sesamin treatment did not induce apoptosis, as seen in the absence of PARP cleavage by Western blotting (Fig. 3A), the measurement of caspase-3/7 activity (Fig. 3B), and negative Annexin V staining (Fig. 3C). Detection of high mobility group box 1 (HMGB1) by immunofluorescence and $\beta$-galactosidase activity assay did not show the presence of necrosis or cellular senescence, respectively (Fig. 3D and E). These results indicate that sesamin-induced loss of cell viability is associated with autophagy in colon cancer cells. 

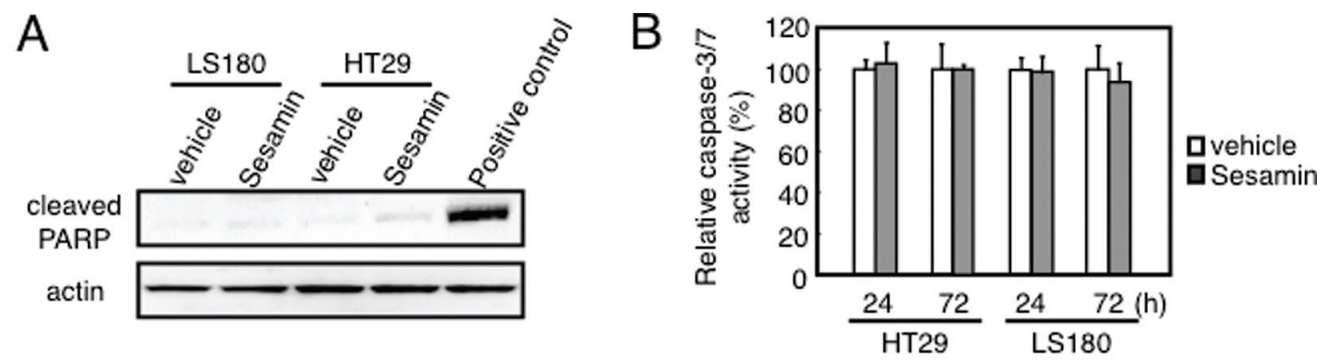

C
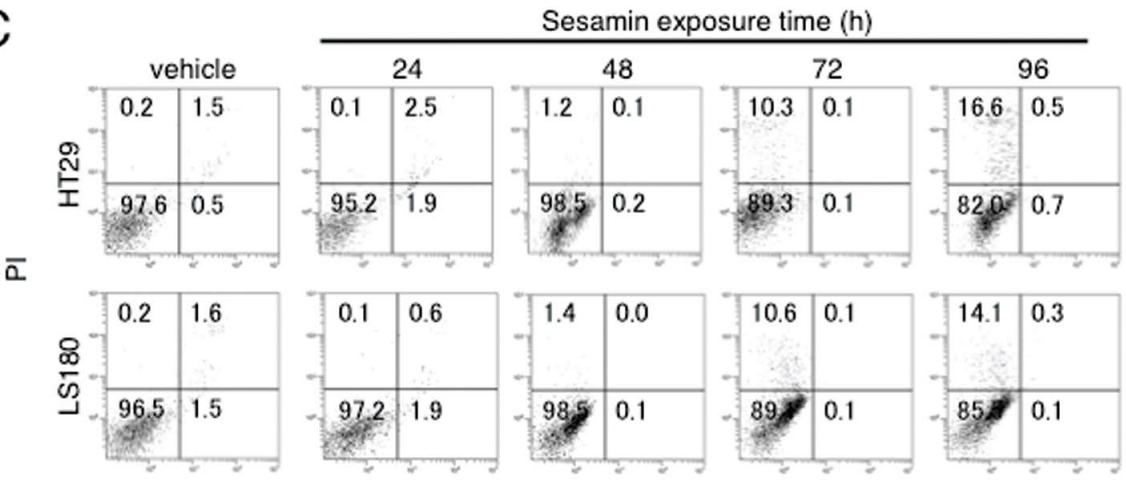

Annexin V FITC

D

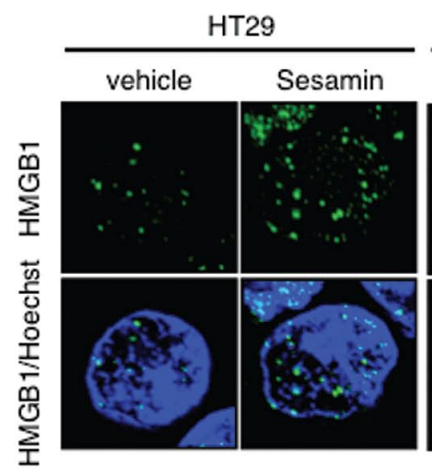

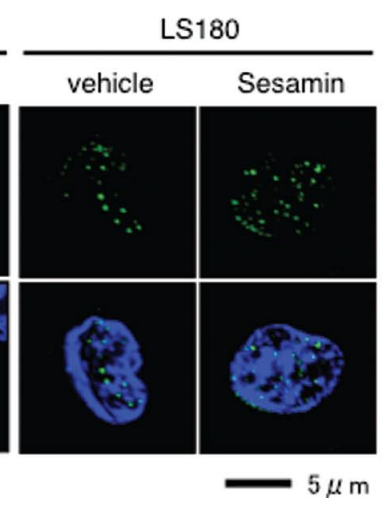

$E$

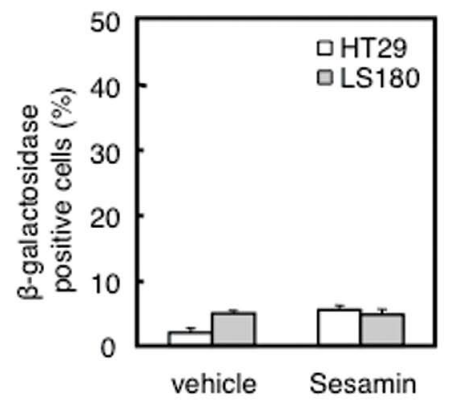

Figure 3. Absence of apoptosis, necrosis, or cellular senescence. (A) LS180 and HT29 cells were treated with or without $50 \mu \mathrm{M}$ sesamin for $72 \mathrm{~h}$ and subjected to Western blot analysis using an antibody against cleaved PARP. A cell lysate of HCT116 cells treated with cisplatin was used as a positive control. (B) HT29 and LS180 cells were seeded in 96-well plates in $100 \mu$ 1 DMEM supplemented with 10\% FBS and incubated with vehicle or $50 \mu$ M sesamin for the times indicated. Caspase-3, and -7 activities were measured and are shown as bar graphs. (C) After treatment of HT29 and LS180 cells with vehicle or $50 \mu \mathrm{M}$ sesamin for the times indicated, apoptotic cells were stained with Annexin V-FITC and PI. (D) HT29 cells treated with vehicle or $50 \mu$ M sesamin were subjected to immunofluorescence using an anti-HMGB1 antibody. Hoechst 33342 was used to counterstain the nuclei. (E) LS180 and HT29 cells were treated with vehicle or $50 \mu \mathrm{M}$ sesamin for 1 week and the senescence-associated $\beta$-Gal activity was measured as described in Materials and methods.

Sesamin reduces phosphorylation of ephrin receptors. Based on a report that the lignan picropodophyllin (PPP) inhibits tyrosine phosphorylation of insulin-like growth factor-1 receptor (IGF1-R) (24), and on the fact that the downstream effector of the Akt/ mammalian target of rapamycin (mTOR) pathway is a regulator of autophagy, the possible effect of sesamin on inhibition of the tyrosine phosphorylation of one of the RTKs was examined. Among 42 RTKs examined, EphA1 and EphB2 showed a decrease in tyrosine phosphorylation after sesamin treatment, with EphB2 showing greater decrease (Fig. 4A and B). Most of the other receptors evaluated showed minimal changes, or an increase in phosphorylation status, including IGF1-R (Fig. 4C).

Silencing of EphA1 and EphB2 inhibits autophagy. To confirm that EphA1 and EphB2 play an essential role in the induction of autophagy, siRNA-mediated silencing of EphA1 and EphB2 was performed followed by treatment of cells with sesamin. siRNA against EphA1 or EphB2 effectively silenced the mRNA expression of these molecules (Fig. 5A). In HT29 cells, silencing of EphA1 or EphB2 was not sufficient to inhibit sesamininduced autophagy (Fig. 5B). In LS180 cells, silencing of EphB2 abrogated the induction of autophagy. As the RTK array showed reduced phosphorylation of both EphA1 and EphB2, we silenced EphA1 and EphB2 in HT29 cells simultaneously. As shown in the Fig. 5B (right panels), simultaneous silencing of EphA1 and EphB2 blocked the induction of autophagy, which was confirmed by Western blotting against LC3. The effect of EphA1 and EphB2 silencing on autophagy inhibition was also confirmed by immunofluorescence using an anti-LC3 antibody (Fig. 5C). These results show that EphA1 and EphB2 play an essential role in sesamin-induced autophagy. 

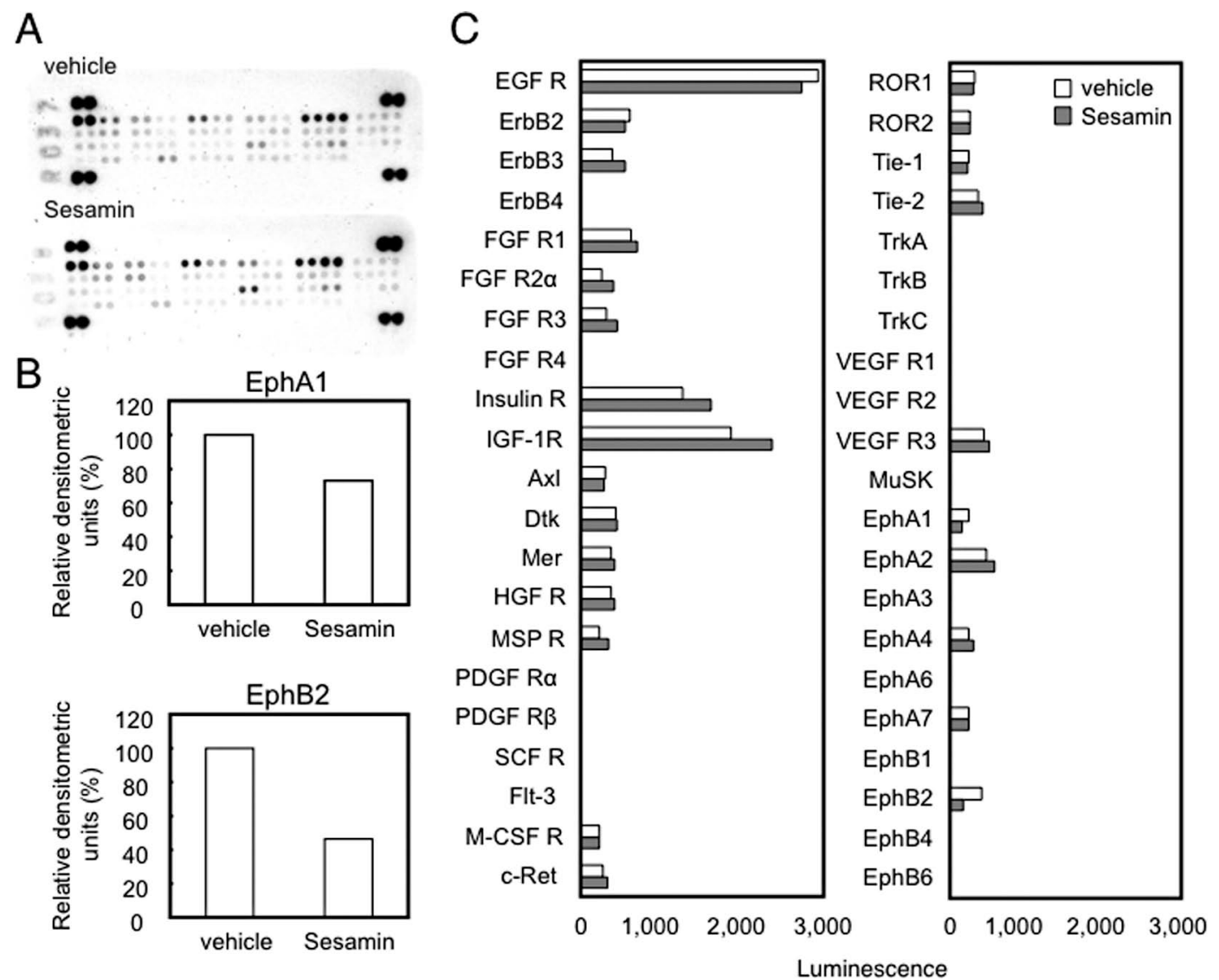

Figure 4. Sesamin inhibits tyrosine phosphorylation of EphA1 and EphB2. (A) HT29 cells were treated with vehicle or $50 \mu \mathrm{M}$ sesamin for $72 \mathrm{~h}$ and subjected to RTK array analysis as described in Materials and methods. Actual blots are shown. (B) Densitometric units of phospho-EphA1 and phospho-EphB2 were measured and are shown as bar graphs. (C) Densitometric units of phospho-RTKs were measured and shown as bar graphs.

Silencing of EphAl and EphB2 counteract sesamin-induced loss of cell viability. To examine whether autophagy is rescuing or harming colon cancer cells from sesamin, the cells were transduced with EphA1 and/or EphB2 siRNAs and the cell viability was tested in sesamin-treated cells. As shown in Fig. 6, silencing of EphA1 or EphB2 opposed the effect of sesamin, with the combination showing the greater effect than the individual silencing. These results show that sesamin-induced autophagy is inhibiting cell viability of colon cancer cells in vitro.

\section{Discussion}

The present study demonstrated that sesamin inhibits cell viability of colon cancer cell lines through a mechanism involving autophagy. In addition, EphA1 and EphB2 play an essential role in the induction of autophagy.

Prior reports suggested that sesamin may inhibit the growth of cancer cells by down-regulating the NF- $\mathrm{KB}$ pathway (5), P-glycoprotein (25), or cyclin D1 (26). The induction of autophagy, found in the present study, is a novel mechanism of sesamininduced inhibition of cancer cell growth.

The finding that sesamin induces autophagy via EphA1 and EphB2 is intriguing because autophagy and EphB2 are considered as tumor suppressors (18). Eph receptors constitute the largest subgroup of RTKs (27). EphB2 is a unique receptor in RTK family because of its tumor suppressor functions. EphB2 maps at chromosome 1p36.1, which is frequently deleted in colorectal cancer (28). EphB2 is frequently mutated in colorectal cancer (29); EphB2 receptor variants are reported in the probands of familial colorectal cancer (30). Expression level of EphB2 decreases with the progress of colorectal lesions in dysplasia-adenoma-carcinoma sequence (31) and the level of EphB2 inversely correlates with prognosis of patients with colorectal cancer $(32,33)$. Furthermore, reduction of EphB2 function accelerates tumourigenesis in the colon and rectum of $\mathrm{Apc}^{\mathrm{Min} /+}$ mice (31). According to previous reports, the best-characterized functions of ephrin-Eph interactions in the intestinal system are the regulation of cell positioning, cell migration and progenitor cell proliferation (34-36). Among these functions, the compartmentalization of tumor cells is thought to be a tumor suppressor function of EphB2 (36). In the present study, silencing of EphB2 abrogated autophagy induced by sesamin, suggesting that the induction of autophagy may be another tumor suppressor mechanism of EphB2. As EphA1 is 53\% identical to EphB2 in the cytoplasmic domain, EphA1 may share functions of EphB2 in the autophagic process.

The effect of autophagy in favoring or interfering with cancer therapeutics is a matter of controversy $(37,38)$. Many anticancer drugs including rapamycin, tamoxifen, and HDAC 
A

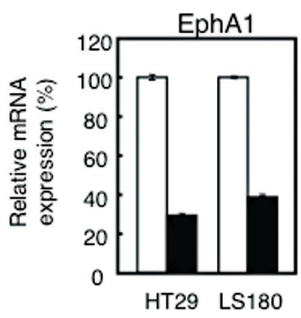

B

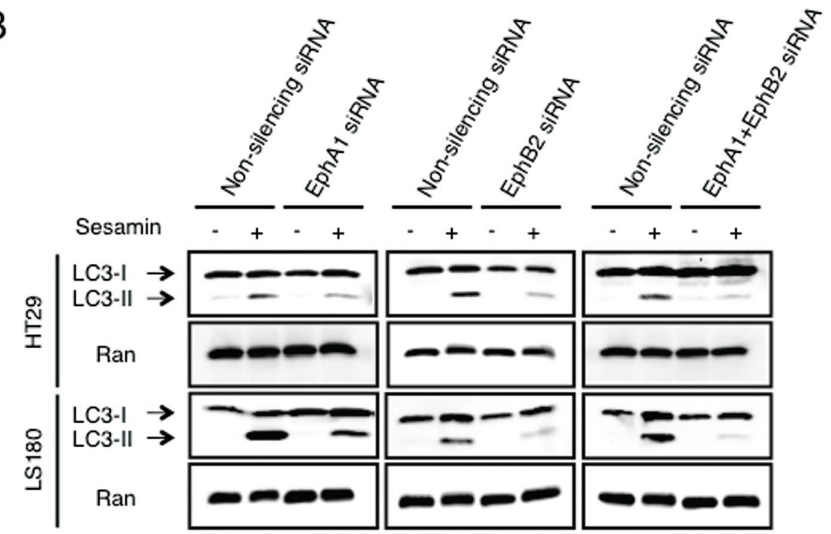

C

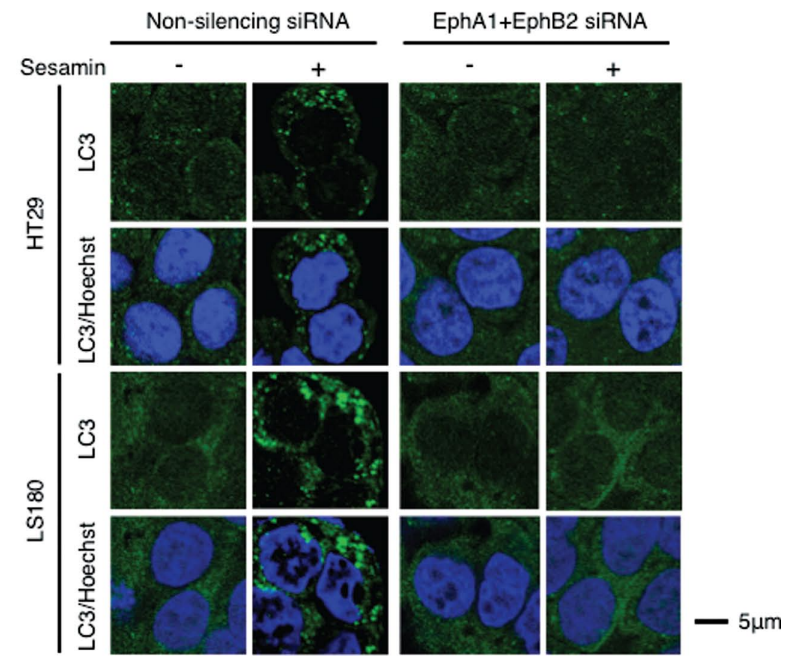

Figure 5. Silencing of EphA1 and EphB2 inhibits autophagy induced by sesamin. (A) A non-silencing control ( $\square$ ) or siRNAs against EphA1 and EphB2 (ם) were transduced into HT29 and LS180 cells, which were then treated with vehicle or $50 \mu \mathrm{M}$ sesamin for $48 \mathrm{~h}$. The levels of EphA1 or EphB2 mRNA were quantified as described in Materials and methods. (B and C) HT29 and LS180 cells were transduced with different sets of siRNAs as indicated in the figure. Cells were treated with vehicle or $50 \mu \mathrm{M}$ sesamin for $48 \mathrm{~h}$ and subjected to Western blot analysis (B) or immunofluorescence analysis (C) using an anti-LC3 antibody.

inhibitors induce autophagy-associated cell death in cancer cells. On the other hand, inhibition of autophagy enhances the effect of cancer therapeutics in many types of malignancies, including colon cancer (39). The present study showed that autophagy induced by sesamin is reducing cell viability of colon cancer cells, at least in vitro. It is still an open question whether autophagy induction by EphB2, and possibly by EphA1, is beneficial or harmful for cancer cells in vivo.

The present result of the RTK array analysis, identifying EphA1 and EphB2 as the only receptors showing reduced phosphorylation levels in sesamin treated cells, is intresting.

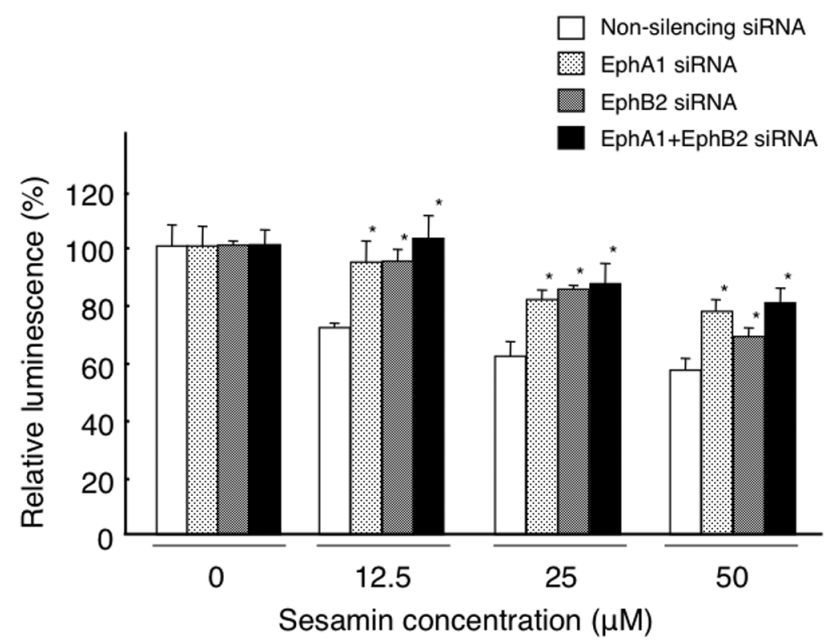

Figure 6. Silencing of EphA1 and EphB2 counteracts sesamin-induced loss of cell viability. Non-silencing control siRNAs, siRNAs against EphA1 and/ or EphB2 were transduced into HT29 cells. Then, the transfectants were treated with different concentrations of sesamin indicated in the figure for $48 \mathrm{~h}$ and the cell viability was measured as described in Materials and methods. * $\mathrm{p}<0.001$ compared to the value of non-silencing control.

Phosphorylation of RTKs indicates activation of the receptors and the downstream Akt pathway, which leads to inhibition of autophagy. However, sesamin-treated cells showed an autophagic phenotype with decreased cell proliferation in vitro. This observation suggests that unphosphorylated EphA1 and EphB2 may overwhelm the signals from the other activated RTKs.

In conclusion, we found that EphA1 and EphB2 acts as regulators of autophagy induced by sesamin. This study provides a novel molecular mechanism for the autophagic process as well as a basis for targeting EphA1 and EphB2 in the chemoprevention of colorectal cancer.

\section{References}

1. Matsumura Y, Kita S, Morimoto S, et al: Antihypertensive effect of sesamin. I. Protection against deoxycorticosterone acetatesalt-induced hypertension and cardiovascular hypertrophy. Biol Pharm Bull 18: 1016-1019, 1995.

2. Noguchi T, Ikeda K, Sasaki Y, et al: Effects of vitamin E and sesamin on hypertension and cerebral thrombogenesis in strokeprone spontaneously hypertensive rats. Hypertens Res 24: 735-742, 2001.

3. Hirose N, Inoue T, Nishihara K, et al: Inhibition of cholesterol absorption and synthesis in rats by sesamin. J Lipid Res 32: 629-638, 1991.

4. Ogawa H, Sasagawa S, Murakami T and Yoshizumi H: Sesame lignans modulate cholesterol metabolism in the stroke-prone spontaneously hypertensive rat. Clin Exp Pharmacol Physiol Suppl 22: S310-S312, 1995.

5. Harikumar KB, Sung B, Tharakan ST, et al: Sesamin manifests chemopreventive effects through the suppression of NF-kappaBregulated cell survival, proliferation, invasion, and angiogenic gene products. Mol Cancer Res 8: 751-761, 2010.

6. Miyahara Y, Komiya T, Katsuzaki H, et al: Sesamin and episesamin induce apoptosis in human lymphoid leukemia Molt 4B cells. Int J Mol Med 6: 43-46, 2000.

7. Levine B and Klionsky DJ: Development by self-digestion: molecular mechanisms and biological functions of autophagy. Dev Cell 6: 463-477, 2004.

8. Klionsky DJ: Autophagy: from phenomenology to molecular understanding in less than a decade. Nat Rev Mol Cell Biol 8: 931-937, 2007. 
9. Mizushima N: Autophagy: process and function. Genes Dev 21: 2861-2873, 2007.

10. Cockle SM and Dean RT: Distinct proteolytic mechanisms in serum-sufficient and serum-restricted fibroblasts. Transformed $3 \mathrm{~T} 3$ cells fail to regulate proteolysis in relation to culture density only during serum-sufficiency. Biochem J 221: 53-60, 1984.

11. Gronostajski RM and Pardee AB: Protein degradation in 3T3 cells and tumorigenic transformed 3T3 cells. J Cell Physiol 119: 127-132, 1984

12. Gunn JM, Clark MG, Knowles SE, Hopgood MF and Ballard FJ: Reduced rates of proteolysis in transformed cells. Nature 266 58-60, 1977.

13. Knecht E, Hernandez-Yago J and Grisolia S: Regulation of lysosomal autophagy in transformed and non-transformed mouse fibroblasts under several growth conditions. Exp Cell Res 154: 224-232, 1984

14. Lockwood TD and Minassian IA: Protein turnover and proliferation. Failure of SV-3T3 cells to increase lysosomal proteinases, increase protein degradation and cease net protein accumulation. Biochem J 206: 251-258, 1982.

15. Canuto RA, Tessitore L, Muzio G, Autelli R and Baccino FM: Tissue protein turnover during liver carcinogenesis. Carcinogenesis 14: 2581-2587, 1993.

16. Kisen GO, Tessitore L, Costelli P, et al: Reduced autophagic activity in primary rat hepatocellular carcinoma and ascites hepatoma cells. Carcinogenesis 14: 2501-2505, 1993.

17. Schwarze PE and Seglen PO: Reduced autophagic activity, improved protein balance and enhanced in vitro survival of hepatocytes isolated from carcinogen-treated rats. Exp Cell Res 157: $15-28,1985$

18. Gozuacik D and Kimchi A: Autophagy as a cell death and tumor suppressor mechanism. Oncogene 23: 2891-2906, 2004.

19. Qu X, Yu J, Bhagat G, et al: Promotion of tumorigenesis by heterozygous disruption of the beclin 1 autophagy gene. J Clin Invest 112: 1809-1820, 2003

20. Yue Z, Jin S, Yang C, Levine AJ and Heintz N: Beclin 1, an autophagy gene essential for early embryonic development, is a haploinsufficient tumor suppressor. Proc Natl Acad Sci USA 100: 15077-15082, 2003.

21. Levine B and Abrams J: p53: The Janus of autophagy? Nat Cell Biol 10: 637-639, 2008

22. Levine B: Cell biology: autophagy and cancer. Nature 446: 745-747, 2007.

23. Munafo DB and Colombo MI: A novel assay to study autophagy: regulation of autophagosome vacuole size by amino acid deprivation. J Cell Sci 114: 3619-3629, 2001.

24. Vasilcanu D, Girnita A, Girnita L, Vasilcanu R, Axelson M and Larsson O: The cyclolignan PPP induces activation loop-specific inhibition of tyrosine phosphorylation of the insulin-like growth factor-1 receptor. Link to the phosphatidyl inositol-3 kinase/Akt apoptotic pathway. Oncogene 23: 7854-7862, 2004.
25. Nabekura T, Yamaki T, Ueno K and Kitagawa S: Inhibition of P-glycoprotein and multidrug resistance protein 1 by dietary phytochemicals. Cancer Chemother Pharmacol 62: 867-873, 2008.

26. Yokota T, Matsuzaki Y, Koyama M, et al: Sesamin, a lignan of sesame, down-regulates cyclin D1 protein expression in human tumor cells. Cancer Sci 98: 1447-1453, 2007.

27. Pasquale EB: Eph receptors and ephrins in cancer: bidirectional signalling and beyond. Nat Rev Cancer 10: 165-180, 2010.

28. Praml C, Finke LH, Herfarth C, Schlag P, Schwab M and Amler L: Deletion mapping defines different regions in 1p34.2-pter that may harbor genetic information related to human colorectal cancer. Oncogene 11: 1357-1362, 1995.

29. Alazzouzi H, Davalos V, Kokko A, et al: Mechanisms of inactivation of the receptor tyrosine kinase EPHB2 in colorectal tumors. Cancer Res 65: 10170-10173, 2005.

30. Zogopoulos G, Jorgensen C, Bacani J, et al: Germline EPHB2 receptor variants in familial colorectal cancer. PLoS One 3: E2885, 2008.

31. Batlle E, Bacani J, Begthel H, et al: EphB receptor activity suppresses colorectal cancer progression. Nature 435: 1126-1130, 2005.

32. Guo DL, Zhang J, Yuen ST, et al: Reduced expression of EphB2 that parallels invasion and metastasis in colorectal tumours. Carcinogenesis 27: 454-464, 2006.

33. Jubb AM, Zhong F, Bheddah S, et al: EphB2 is a prognostic factor in colorectal cancer. Clin Cancer Res 11: 5181-5187, 2005.

34. Pasquale EB: Eph-ephrin bidirectional signaling in physiology and disease. Cell 133: 38-52, 2008

35. Holmberg J, Genander M, Halford MM, et al: EphB receptors coordinate migration and proliferation in the intestinal stem cell niche. Cell 125: 1151-1163, 2006

36. Cortina C, Palomo-Ponce S, Iglesias M, et al: EphB-ephrin-B interactions suppress colorectal cancer progression by compartmentalizing tumor cells. Nat Genet 39: 1376-1383, 2007.

37. Kondo Y, Kanzawa T, Sawaya R and Kondo S: The role of autophagy in cancer development and response to therapy. Nat Rev Cancer 5: 726-734, 2005.

38. Hippert MM, O'Toole PS and Thorburn A: Autophagy in cancer: good, bad, or both? Cancer Res 66: 9349-9351, 2006.

39. Li J, Hou N, Faried A, Tsutsumi S and Kuwano H: Inhibition of autophagy augments 5-fluorouracil chemotherapy in human colon cancer in vitro and in vivo model. Eur J Cancer 46: 1900-1909, 2010. 